\title{
1 Acute aggressive behavior perturbates the oxidative status of a wild 2 bird independently of testosterone and progesterone
}

3 Lucia Mentesana ${ }^{1 *} \&$ Nicolas M. Adreani ${ }^{2,3}$

$4 \quad{ }^{1}$ Evolutionary Physiology Research Group, Max Planck Institute for Ornithology, Seewiesen, Germany

$5{ }^{2}$ Department of Behavioural Neurobiology, Max Planck Institute for Ornithology, Seewiesen,

6 Germany

$7 \quad{ }^{3}$ Konrad Lorenz Research Centre for Behavioural and Cognitive Biology, University of Vienna,

8 Grünau im Almtal, Austria

$9 *$ Author for correspondence (lmentesana@ orn.mpg.de)

\section{Abstract}

11 Aerobically demanding activities like aggression can lead to an elevated oxidative 12 metabolism affecting the concentration of pro-oxidant and antioxidant compounds and can 13 result in an overall perturbation of the oxidative status. Aggression may also alter the 14 oxidative status indirectly through an increase in testosterone and progesterone 15 concentrations. Given that changes in the oxidative status could represent a physiological cost 16 of aggression, we tested the hypothesis that acute conspecific aggression impairs the oxidative 17 status and evaluated the role of testosterone and progesterone as potential mediators. To 18 achieve this, we experimentally manipulated the aggressive behavior of wild female and male 19 birds and measured the concentrations of pro-oxidants, enzymatic- and non-enzymatic 20 antioxidants, testosterone and progesterone in blood. After 20 minutes of conspecific 21 aggressive behavior, both sexes had lower concentrations of non-enzymatic antioxidants than 22 control individuals. This effect was independent of testosterone and progesterone 23 concentrations, and much stronger in females than in males. Further, only in females (but not 24 in males) being more aggressive came at the expense of lower antioxidant concentration. We 25 provide the first experimental evidence that acute aggressive behavior perturbates the 26 oxidative state of a wild vertebrate independently of testosterone and progesterone, with 27 potential ecological and evolutionary implications given the role of the redox system in shaping life-history traits.

30 Keywords: female aggression, male aggression, redox system, territoriality, rufous 


\section{1. Introduction}

33 Animals frequently engage in aggressive behaviors to obtain and secure limited resources that 34 can enhance their reproductive success, such as a breeding territory (Beckman \& Ames, 1998; 35 Duckworth, 2006; Demas et al. 2007; Rosvall, 2008; Smith \& Blumstein 2008; Clutton36 Brock, 2009). However, aggressive behaviors entail costs (e.g., territory loss or injuries), 37 which could partially explain why individuals differ in their degree of aggressiveness. A 38 particularly relevant but understudied cost associated with this behavior concerns the oxidative state of an individual (Costantini et al., 2008; Isaksson et al., 2011). The oxidative state of an individual is determined by the concentration of pro-oxidants (i.e., reactive oxygen species) and antioxidants (i.e., non-enzymatic and enzymatic compounds) present in cells and tissues (reviewed by Costantini 2019). A change in any of these molecular components in favor of pro-oxidants can lead to damage of biomolecules such as lipids, proteins and DNA (reviewed by Costantini 2008; Monaghan et al., 2009). This damage can shape life-history decisions of individuals as well as life history traits such as reproduction and longevity (e.g., Finkel and Holbrook, 2000; Costantini 2008; Monaghan et al. 2009), potentially translating into ecological and evolutionary consequences.

Aggressive behaviors are energetically demanding activities that increase the metabolic rate, exposing an individual to an elevated concentration of pro-oxidants (Figure 1a; Costantini 2008; Powers \& Jackson, 2008; Skrip \& McWilliams, 2016; Cooper-Mullin \& McWilliams, 2016; but see Salin et al., 2015). Such perturbations in the oxidative status of animals are expected since more than $90 \%$ of the cellular energy is generated by the mitochondria (Bottje 2015) and natural by-products of aerobic respiration are reactive oxygen species (ROS). However, our understanding of aggression altering the oxidative state is still in its early stages. In selected lines of mice (Mus musculus domesticus), aggressive males had lower levels of antioxidants than non-aggressive males (Costantini et al., 2008). In contrast, aggressive males of wild-caught white skinks (Egernia whitii) had higher levels of nonenzymatic antioxidants than less aggressive males, whereas in females no such relationship was apparent (Isaksson et al., 2011). Acute aggressive behaviors, such as territorial fights, that represent an increase in energy expenditure, could also incur an oxidative challenge; yet it remains unknown whether this is the case.

The oxidative state of an individual can be indirectly altered by aggressive behaviors through an increase in steroid hormone concentrations (Figure 1b; Schantz et al., 1999; Alonso-Alvarez et al., 2007). The sex steroid testosterone is assumed to be the key hormone related to resource-defense aggression and can increase during aggressive interactions in males (Wingfield et al. 1990, Hirchenhauser et al., 2006; Goymann et al., 2007; Hau 2007). Testosterone has been proposed to be a cause of increased ROS production (e.g., Alonso-

68 Alvarez et al., 2007, Koch et al. 2016), which can occur either because testosterone enhances 
the metabolic rate of individuals (e.g., Marler \& Moore, 1989; Welle et al. 1992; Wikelsky et al., 1999; Buchanan et al. 2001, Koch et al. 2016) and/or because testosterone has intrinsic oxidative properties (e.g., Zhu et al., 1997; Chainy et al., 2009; Casagrande et al. 2011). An increase in aggressive behavior after a territorial intrusion can, therefore, potentially lead to an increase in testosterone concentrations (e.g., Wingfield \& Wada 1989; Wingfield \& Hahn, 1994; McGlothlin et al., 2008), with testosterone levels influencing the concentrations of prooxidant and antioxidant compounds and resulting in an overall perturbation of the oxidative state. To date, only one study has looked at the relationship between aggression, oxidative status and testosterone concentrations in a wild-caught vertebrate (Isaksson et al., 2011). Isaksson et al (2011) found that the aggressive phenotype of male white skinks, but not testosterone concentrations, was positively related to the oxidative condition of individuals. Aggression can also be associated with another steroid hormone: progesterone. Progesterone has been mainly proposed as a mediator of aggressive behavior in females (e.g., Goymann et al., 2008; but see e.g., Adreani et al., 2018). As with testosterone, progesterone can increase the metabolic rate of organisms (Gavrilova-Jordan \& Price 2007) and the concentration of pro-oxidants (only from studies done in vitro e.g., Zhu et al., 1997; Itagaki et al., 2005). Investigating the relationships between aggressive behavior, oxidative status, testosterone and progesterone in females and males is fundamental if we aim to understand the sex-specificity of these physiological pathways.

The main goal of our study was to test the hypothesis that an acute increase in conspecific aggressive behavior can impair the oxidative status of wild rufous horneros (Aves: Furnarius rufus, hereafter termed hornero), and to test whether this was related to testosterone and progesterone concentrations. To elicit an aggressive response, we challenged female and male birds with 20 minutes of simulated conspecific territorial intrusions (STI) during the nest-building period. Immediately after the STI we collected blood samples to measure the concentrations of three oxidative status markers, testosterone and progesterone. We then compared these concentrations with the ones of birds that did not engage in aggressive behaviors. The hornero represents an excellent model system to study the effects of acute aggression across sexes. It is a seasonal breeder, and both females and males are involved in territorial defense throughout the year (Fraga, 1980; Diniz et al., 2016; Mentesana et al. 2020). Further, horneros are sexually monomorphic in plumage coloration and body size (i.e., there is no difference in body condition between sexes; Diniz et al., 2016), and all breeding behaviors studied so far, except for aggressive behaviors, are shared and coordinated between the sexes (Massoni et al., 2012; Diniz et al. 2018; Mentesana et al. 2020). 
103

104

105

106

107

108

109

110

111

112

113

114

115

116

117

118

120

121

122

123

124

125

126

127

128

129

(a)
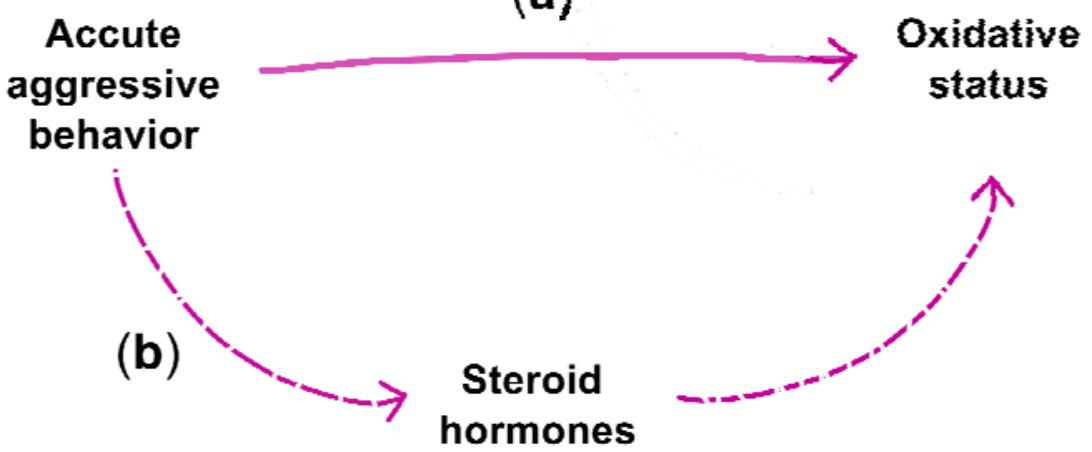

Figure 1. Hypothesized pathways by which acute aggressive behaviors can perturbate the individuals' oxidative status: (a) directly through an increase in the metabolic rate, and/or (b) indirectly through the action of steroid hormones.

\section{Materials and methods}

\subsection{Field site and simulated territorial intrusion (STI)}

The study was conducted between August 22nd and September 28th of 2016 on the campus of INIA 'Las Brujas' (National Institute of Agricultural Research), Department of Canelones, Uruguay (344ㅇ' S, 56 20 ' W; 0-35 m a.s.l.). During this period, horneros were building their nests and females were close to egg laying. In total, we collected behavioral and physiological data from birds defending 51 territories that were subjected to the experimental treatments between 7 am and $1 \mathrm{pm}$.

We experimentally manipulated the hornero's aggressive behavior by performing simulated territorial intrusions (STI). For each territory, the treatment (i.e., control or STI) was randomly determined. STI is a common method to investigate the aggressive and physiological responses of a territory holder in response to an intruder (e.g., Gill et al., 2007; Apfelbeck \& Goymann, 2011; Villavicencio et al., 2014), and STIs are known to be effective in horneros (Adreani et al., 2018; Mentesana et al., 2020). In particular, a stuffed decoy of a male hornero, a speaker and folded mist nets were placed $5 \mathrm{~m}$ away from the nest of focal pairs, and solo songs and duets of conspecifics were played (recordings were extracted from the database www.xeno-canto.org and the amplitude of every recording was normalized). For ethical reasons we could not use more than one decoy (i.e., the visual component of the STIs was the same for every territory). However, horneros are sexually indistinguishable in morphological traits (i.e., mass, tarsus and bill) and plumage coloration (assessed in relation to the avian visual system; Diniz et al., 2016). Further, in a comparable STI study done in European great tits (Parus major) the decoys used $(\mathrm{n}=15)$ did not explain any significant 
130 variation in aggressive behaviors (Araya-Ajoy et al., 2013); thus, suggesting that the use of 131 only one decoy did not bias our results. On the other hand, given the lack of information on 132 the function of the different vocal signals in horneros, we decided to form a pool of ten male 133 solo songs and ten duets to ensure that both females and males responded to the STI. We also 134 added ten 'silence' files of 7-15 sec duration to have unpredictable gaps between the different 135 stimuli. The auditory stimuli for each STI were randomly selected from this pool to avoid 136 pseudo-replication of the acoustic component across territories (e.g., Apfelbek et al., 2011). 137 Horneros are suboscines and do not learn their vocalizations (Freeman et al., 2017); compared 138 to oscines, the acoustic variability of songs and duets across individuals is low (Freeman et 139 al., 2017). Hence, it is likely that all playbacks elicited a comparable behavioral response 140 across territories despite the auditory stimuli being randomly chosen from the pool.

141 Challenged birds (hereafter 'STI birds', $\mathrm{N}_{\text {females, }} \mathrm{N}_{\text {males }}=9,18$ ) were exposed to a 142 simulated territorial intrusion by a conspecific for 20 minutes. We decided on this 143 experimental time because, in birds, plasma levels of testosterone increase after 10 minutes of 144 the onset of the challenge and can remain high until an hour after onset of the intrusion 145 (Wingfield \& Wada 1989). During the 20 minutes of territorial intrusion, two observers 146 recorded the aggressive behavior of the territorial couple in digital voice recorders (Philips 147 VoiceTraicer DVT1200 and Olympus Digital Recorder VN-733 PC). Each observer followed 148 one member of the focal STI pair. Which individual of the pair to follow was randomly 149 determined prior to the experimental trial. Sex identification in the field was done based on 150 the singing behavior (i.e., the contribution of females and males in the duets are acoustically 151 distinct from each other; Laje \& Mindlin 2003) and was later confirmed by molecular sexing 152 (for details see Adreani et al., 2018). Observers did not differ in their quantification skills for 153 the behaviors scored (Figure S1; Table S1; see Supplementary Material section for a detailed 154 description of the inter-observer reliability test). The following parameters were recorded: 1) 155 response latency (time between start of playback and first approach), 2) time spent within $5 \mathrm{~m}$ 156 of the decoy, 3) time spent on the nest, 4) number of solo songs, 5) number of duets, and 6) 157 number of flights over the decoy (which represent both attacks and attempted attacks towards 158 the decoy). After 20 minutes, mist nets were unfolded for a maximum of ten additional 159 minutes. Capturing horneros passively is of great difficulty (LM \& NMA, personal 160 observations). Therefore, to attract control individuals $\left(\mathrm{N}_{\text {females, }} \mathrm{N}_{\text {males }}=7,24\right)$ we used a 161 stuffed decoy of a male hornero and playbacks, but mist nets were kept open since the 162 beginning of the stimuli presentation. That is, control birds did not perform aggressive 163 behaviors since they were caught as soon as they approached the experimental set-up. Control 164 birds were captured within the first minutes of exposure to the decoy and playback (mean 165 capture time $\pm \mathrm{sd}=4.57 \pm 2.97$ mins for females and $4.50 \pm 3.75 \mathrm{mins}$ for males), while STI 166 birds were captured after 20 minutes of exposure (mean capture time $\pm \mathrm{sd}=23.11 \pm 2.33 \mathrm{mins}$ 
167 for females and $25.94 \pm 4.68$ mins for males; Figure S2). From all captured birds, $80 \mu 1$ of 168 blood were taken from the brachial vein using heparinized capillaries. In addition, 169 morphometric measurements (i.e. mass, tarsus length and wing length) were recorded. 170 Control and STI individuals did not differ in body condition index (Table S2). Before 171 releasing them back in their territories, each individual was marked with a numbered aluminum ring and a unique combination of three colored plastic split rings.

174 the decoy (Adreani et al., 2018; Mentesana et al., 2020). In our experiments STIs elicited

175 similar behavioral responses as intrusions by real conspecifics or territorial conflicts with 176 neighbours (LM \& NMA, personal observations). Females and males showed clear behavioral 177 responses towards our treatment. However, once the male was captured, the female would 178 immediately stop any territorial behavior and start alarm calling. This explains why, during 179 our experiments we caught more than twice the number of males compared to females, and 180 why we generally caught only one member of the pair.

\subsection{Oxidative status analyses}

183 The oxidative status of female and male horneros was determined from both non-enzymatic 184 and enzymatic antioxidants present in plasma and red blood cells respectively, and from reactive oxygen species present in plasma. Non-enzymatic antioxidant concentrations (OXY) were measured using the OXY-Adsorbent test (Diacron International SRL, Grosseto, Italy;

187 Costantini et al. (2006). This assay quantifies the ability of antioxidants to cope with the 188 oxidant action of hypochlorous acid. Enzymatic antioxidant concentrations of glutathione 189 peroxidase (GPX) were determined using the Ransel assay (Randox Laboratories, Germany; 190 Costantini et al. 2011). This assay determines the activity of the enzyme when catalyzing the 191 oxidation of glutathione by cumune hydroperoxide. Oxidative damage was quantified by measuring the concentrations of reactive oxygen metabolites (ROMs) with the d-ROM test (Diacron International SRL, Grosseto, Italy; Costantini et al. 2006). ROMs are end-products of free radical reactions with biological macromolecules. The absorbance was read using a spectrophotometer (Thermo Scientific Multiskan GO) at a wavelength of $546 \mathrm{~nm}$ for OXY and ROMs (endpoint mode) and $340 \mathrm{~nm}$ for GPX (kinetic modality).

\subsection{Testosterone and progesterone analyses}

199 Testosterone and progesterone hormones were extracted from plasma, and concentrations 200 were determined by radioimmunoassay following Goymann et al. (2008). Females and males 201 were analyzed in two separate assays because the samples collected for males were also part 202 of a complementary study (for details see Adreani et al. 2018). In females, mean recoveries 203 following extractions of the samples were (mean \pm sd) $89 \pm 2 \%$ and $80 \pm 3 \%$ for testosterone 
204 and progesterone, respectively. The lower detection limits of the testosterone and

205 progesterone assay were 0.39 and $0.38 \mathrm{pg} /$ tube. For both hormones, all samples used in our

206 analyses were above these detection limits. The intra-assay variation, calculated from of an

207 extracted chicken pool, were $7.5 \%$ for testosterone and $12.2 \%$ for progesterone. It is worth

208 noting that, despite analyzing female and male samples in separate assays, mean recoveries

209 for both hormones were similar between sexes (male mean recovery of testosterone $\pm \mathrm{sd}=87$

$210 \pm 1.9 \%$ and progesterone $=84 \pm 5$ ). It is therefore unlikely that hormonal differences between

211 females and males are due to samples measured in separate assays.

\subsection{Statistical analysis}

214 All our analyses were performed using the R-packages "lme4" and "arm" in R-3.3.3 (R Core

215 Team, 2013) in a Bayesian framework with non-informative priors. For all models, we

216 assumed a Gaussian error distribution, and model fit was assessed by visual inspection of the

217 residuals. We used the "sim" function to simulate posterior distributions of the model

218 parameters. Based on 10,000 simulations, we extracted the mean value and $95 \%$ credible

219 intervals (CrI) (Gelman \& Hill, 2007). Assessment of statistical support was obtained from

220 the posterior distribution of each parameter (Korner-Nievergelt et al., 2015). We considered

221 an effect to be statistically meaningful when the posterior probability of the mean difference

222 (hereafter termed ' $p$ (dif.)') between compared estimates was higher than $95 \%$ or when the

223 estimated CrI did not include zero. For details on this approach see Korner-Nievergelt et al.

224 (2015).

225 First, we ran five linear models fitting OXY, GPX, ROMs, testosterone and progesterone

226 as response variables to i) compare the oxidative status, testosterone and progesterone

227 concentrations between female and male control birds (i.e., individuals that better represent

228 the baseline status), and to ii) study the effect of 20 minutes of aggressive behavior on the

229 oxidative status, testosterone and progesterone concentrations of female and male horneros.

230 To do so, sex (female vs male) and treatment (control vs STI) were included as fixed effects,

231 as well as the interaction between both factors. We also included capture time and handling

232 time as covariates to account for the possible variation that they might add (see Table S3).

233 Preliminary exploration of our data showed that time of the day as well as observer ID did not

234 explain significant variance; thus, to avoid overparameterization, they were not included in

235 the final model.

236 Second, we ran three linear models to investigate the relationship between oxidative 237 status and testosterone concentrations in control individuals and three similar models to 238 investigate the relationship between oxidative status and progesterone. In the first set of 239 models OXY, GPX or ROMs were fitted as response variables and testosterone as 240 explanatory variable. In the second set of models, progesterone was fitted as explanatory 
241 variable. In all models, sex and the interaction of sex with the hormonal levels were included

242 as fixed factors. Since females and males differ in their testosterone concentrations (see

243 Figure 1), for each sex we normalized the hormonal levels between 0 and 1 . This allowed us

244 to compare results between sexes.

245 Finally, within the STI birds, we studied the effect that aggressive behavior had on the

246 oxidative status, testosterone and progesterone concentrations. To do so, we fitted OXY,

247 GPX, ROMs, testosterone and progesterone concentrations as the response variable in

248 separate linear models. While GPX and ROMs were square-root transformed for a better fit of

249 each model, testosterone was log-transformed for the same purpose. Although we measured 6

250 behavioral responses as proxies of aggressiveness (detailed above), to avoid multiple testing,

251 here we only used "flights over the decoy" as explanatory variable. We followed this

252 approach based on a recent study that shows that in horneros the 6 behavioral responses are to

253 some extent correlated, but "flights over the decoy" is the best proxy for conspecific territorial

254 aggression during the breeding season (Mentesana et al. 2020). Male horneros are more

255 aggressive than females (Diniz et al. 2018; Mentesana et al. 2020); thus, for each sex, we

256 normalized the behavioral variables between 0 and 1 in order to capture the aggression

257 gradient in each sex and to be able to compare the effects between the sexes in one model.

258 Sex and the interaction of this variable with aggressive behavior were fitted as fixed factors.

259 Given that we had a limited sample size and that neither capture nor handling time had an

260 effect on any of the oxidative parameters or testosterone levels (see Table S3), these two

261 variables were not included in the final models.

262 


\section{3. Results}

264

265

266

267

268

269

270

271

272

273

274

275

276

277

278

279

280

281

282

3.1 Sex differences in the concentrations of oxidative markers, testosterone and progesterone of control birds

Control females had a lower non-enzymatic antioxidant capacity (OXY) than control males (Figure 2A; $p$ (dif.) > 99.99\%, Table S3). Both sexes had similar enzymatic antioxidant concentrations (GPX; Figure 2B; $p($ dif. $)=79.03 \%$, Table S3). We found a trend for control males having lower levels of pro-oxidants than females (ROMs; Figure 2C; $p$ (dif.) $=94.67 \%$, Table S3). Control females had lower testosterone concentrations than control males (Figure 2D; $p($ dif. $)=99.86 \%$, Table S3), but we found no difference in progesterone concentrations between sexes (Figure 2E; $p($ dif. $)=62.51 \%$, Table S3). There was no relationship between any oxidative marker and testosterone or progesterone concentrations in either sex (Figure 3, Table S4 Table S5).

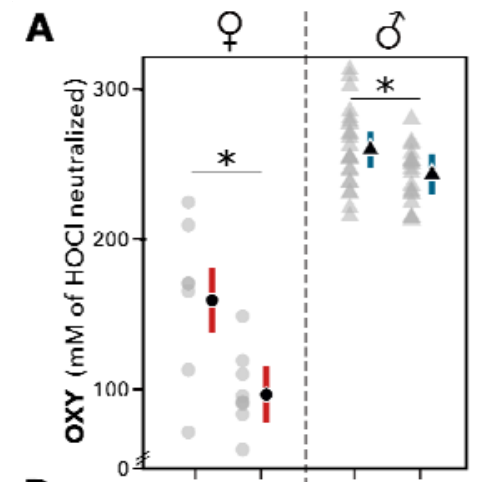

D

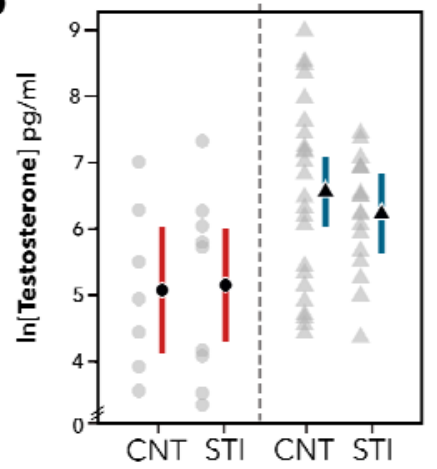

B

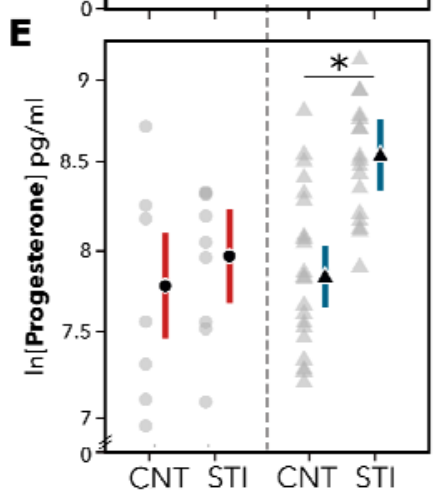

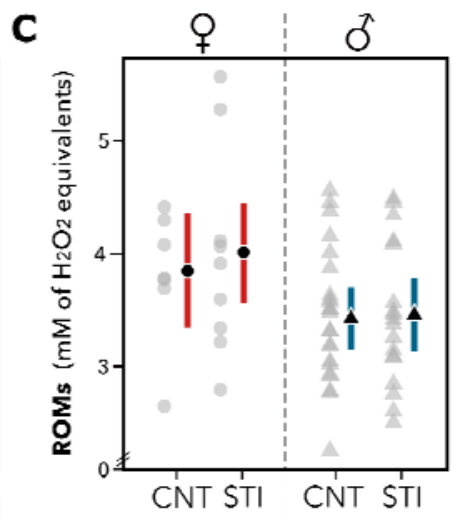

Figure 2. Non-enzymatic antioxidants (OXY), (B) enzymatic antioxidants (GPX), (C) pro-oxidants (ROMs), (D) testosterone and (E) progesterone concentrations measured in control (CNT) and simulated territorial intrusion (STI) female $(\uparrow)$ and male $(\precsim)$ horneros. Grey circles and triangles show the individual raw data for females and males, respectively. Mean estimates of the model are indicated with black symbols, and 95\% credible intervals are represented with colored vertical bars. Statistically meaningful differences are marked with an asterisk. 

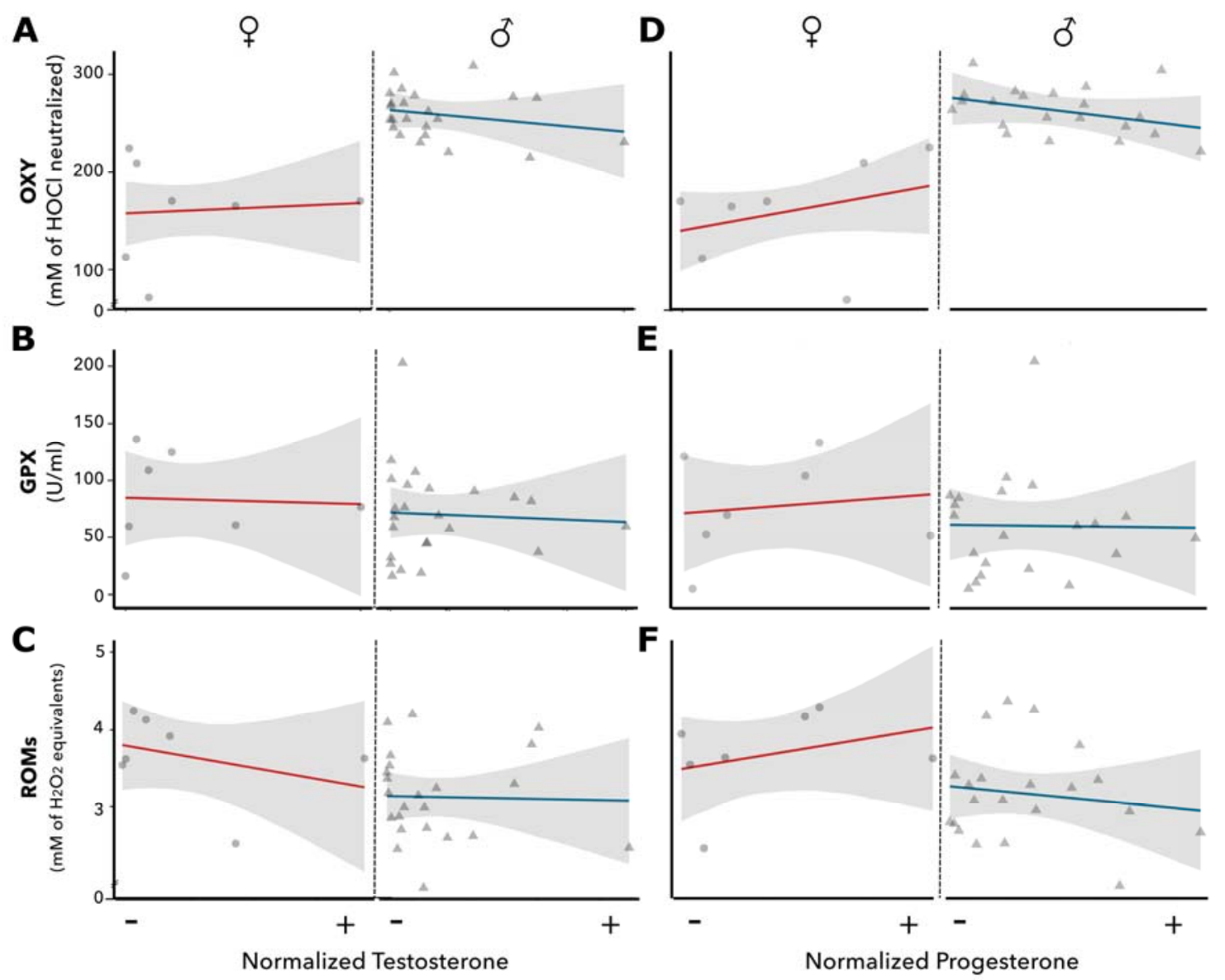

Figure 3. Relationships between the concentrations of non-enzymatic antioxidant (OXY), enzymatic antioxidant (GPX) and pro-oxidants (ROMs) with testosterone (A-C) and progesterone (D-F) in control female $(\uparrow)$ and male $(\widehat{)})$ horneros. For each sex, testosterone concentrations were normalized between 0 and 1 . Grey circles and triangles show the raw individual data for females and males, respectively. Mean estimates of the model are indicated with colored straight lines, and $95 \%$ credible intervals are represented with grey ribbons. There is no relationship between any of the oxidative status markers and testosterone or progesterone concentrations.

\subsection{Effect of acute conspecific aggressive behavior on the concentrations of oxidative} markers, testosterone and progesterone

294 Both sexes had lower levels of OXY after 20 minutes of simulated territorial intrusion 295 compared to control individuals (Figure $2 \mathrm{~A} ; p(\text { dif. })_{\text {females }}>99.99 \% ; p(\text { dif. })_{\text {males }}>99.99 \%$ ).

296 However, the treatment affected females and males differently: the difference in OXY concentrations between control and STI was much stronger in females than in males (females' OXY differed by $39.62 \%$ between treatments, while in males this difference was $6.18 \%$; $p($ dif. $)=99.50 \%)$. We found no difference in GPX $\left(p(\text { dif. })_{\text {females }}=76.62 \% ; p(\text { dif. })_{\text {males }}=\right.$ $44.85 \%)$ or ROMs $\left(p(\text { dif. })_{\text {females }}=31.35 \%\right.$; $\left.p(\text { dif. })_{\text {males }}=44.14 \%\right)$ concentrations between 
301 experimental groups (Figure 2 B-C). In relation to steroid hormones, testosterone

302 concentrations did not differ between control and STI groups $\left(p(\text { dif. })_{\text {females }}=45.56 \%\right.$;

$303 p(\text { dif. })_{\text {males }}=79.65 \%$; Figure $2 \mathrm{D}$ ), whereas, only in males, progesterone concentrations of STI

304 males were higher than that of control males $\left(p(\text { dif. })_{\text {females }}<60.5 \% ; p \text { (dif. }\right)_{\text {males }}<99.99 \%$;

305 Figure 2E). For details on model estimates see Table S3.

306

3.3 Relationships between acute conspecific aggressive behavior and the concentrations of oxidative markers, testosterone and progesterone

309 More aggressive females had lower concentrations of antioxidants than less aggressive 310 females (Figure 4A; posterior probability for the slope being < 0: 99.64\%), whereas in males, 311 aggressive behavior and OXY concentrations were unrelated (Figure 4A; posterior probability 312 for the slope being < 0: 52.42\%). For both sexes, aggression levels were unrelated to GPX, 313 ROMs, testosterone (Figure 4B) and progesterone (Figure 4C) concentrations (i.e., for each 314 variable, the posterior probability for the slope being < 0 was smaller than $90 \%$ ). For details on model estimates see Table S6.
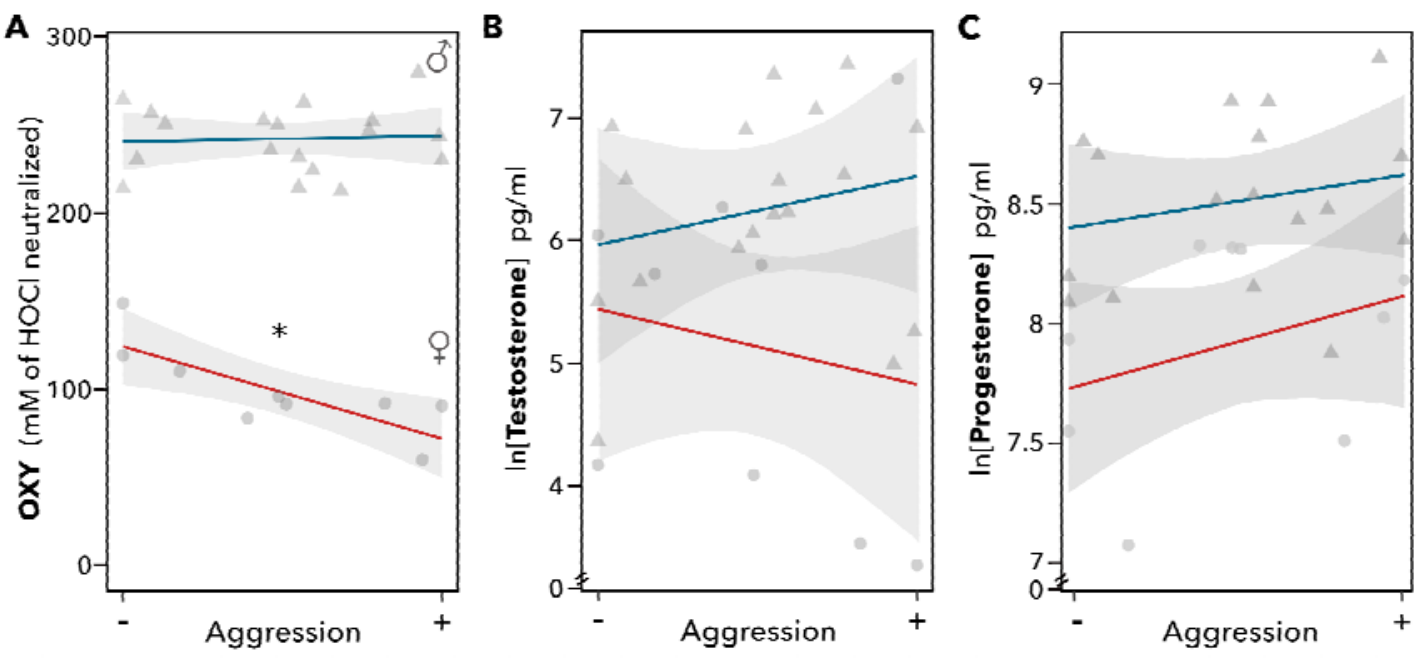

Figure 4. Relationships between the concentrations of (A) non-enzymatic antioxidant (OXY), (B) testosterone and $(\mathrm{C})$ progesterone concentrations with conspecific aggressive behavior in female $(q)$ and male $(\delta)$ horneros. Circles and triangles show the individual raw data for females and males, respectively. Mean estimates of the model are indicated with solid lines ( $q$ : red line; $\delta$ : blue line), and $95 \%$ credible intervals are indicated with a shaded area. Statistically meaningful effects are marked with an asterisk.

\section{Discussion}

326 We experimentally tested if conspecific aggressive behavior induced an acute change in the oxidative state of female and male wild birds, and whether this effect was mediated by 
328

329

330

331

332

333

334

335

336

337

338

339

340

341

342

343

344

345

346

347

348

349

350

351

352

353

354

355

356

357

testosterone or progesterone. Our results provide the first evidence that an acute increase in conspecific aggressive behavior (i.e., for 20 mins) perturbates the oxidative status of an individual by decreasing the concentration of non-enzymatic antioxidants in plasma. This perturbation in oxidative condition was not related to the concentrations of testosterone or progesterone and was much stronger in females than males. Moreover, lower antioxidant concentrations were correlated to higher aggressive behavior in females, but not in males.

\subsection{Sex differences in the concentrations of oxidative markers, testosterone and} progesterone in control birds

Control females had lower concentrations of non-enzymatic antioxidants (OXY) and a trend for higher concentrations of reactive oxygen species (ROMs) than control males (Figure 2A $\& 2 \mathrm{C}$ ). These results were unexpected given that female horneros are less aggressive (Diniz et al. 2018; Mentesana et al., 2020), have lower concentrations of testosterone (Figure 2D) and have similar body condition (Table S2) than males. Also, estrogens, which are generally present at higher concentrations in females close to egg laying compared to males, can increase antioxidant defenses (Halifeoglu et al., 2003). One possible explanation for the sex difference in oxidative status might be that females and males face different reproductive challenges during the early reproductive period (i.e., when females are about to lay eggs). In birds, the resting metabolic rate of females can increase by up to $27 \%$ during the period of egg production (Nilsson \& Råberg, 2001). Such an increase in metabolic rate could potentially explain the females' lower levels of OXY and the higher levels of ROMs ( $p($ dif. $)=$ 94.75\%; Figure 2C) compared to males. The egg laying period is also physiologically demanding since some of the compounds that mothers allocate into their eggs are limited in their availability (Møller et al., 2000; Surai 2002; Hulbert \& Abbott, 2011). Antioxidants, such as vitamin E and carotenoids, cannot be synthesized de novo (e.g., Surai et al., 2001) and so are of dietary origin. Therefore, another possible and non-exclusive explanation for the difference in the oxidative state between sexes, could be associated with females allocating non-enzymatic antioxidant components into the egg yolk (Royle et al., 1999; Mentesana et al., 2019) to protect the developing embryo (Surai, 2002).

\subsection{Effect of conspecific aggressive behavior on oxidative status}

Because reactive oxygen species are a primary product of aerobic metabolism, it is often assumed that behaviors that increase metabolic rate, such as aggression, produce higher rates of reactive oxygen species (Costantini 2008; Powers \& Jackson, 2008; Skrip \& McWilliams, 2016; Cooper-Mullin \& McWilliams, 2016). However, we found no difference in ROMs between control and STI individuals in either sex (Figure 2C). In contrast, an acute increase in aggressive behavior decreased the antioxidant capacity in male and female horneros (Figure 
365 2A): the concentration of OXY was lower in STI than in control birds. These results have two

366 possible explanations: horneros were able to avoid oxidative damage (as measured by ROMs)

367 by depleting non-enzymatic antioxidants or horneros remobilized non-enzymatic antioxidants

368 among tissues (see below). The concentration of enzymatic antioxidants (GPX) did not differ

369 between experimental groups. GPX concentrations increase with behaviors that associated to

370 increased metabolic rate and are sustained over time (e.g., low-medium endurance training or

371 parental provisioning, Powers and Jackson, 2008; Casagrande \& Hau 2018). Such behaviors

372 performed over short-term periods of time can also induce di novo protein synthesis at the

373 cellular level; however, protein synthesis occurs in the time scale of hours (e.g., Hernandez et

374 al. 2000). It is thus possible that the upregulation of GPX remained undetectable after 20

375 minutes of acute aggressive interactions.

376 Aggressive behaviors can also perturbate the oxidative status indirectly through an increase in steroid hormone concentrations (Schantz et al., 1999; Alonso-Alvarez et al., 2007;

378 Isaksson et al., 2011). However, our results suggest that neither testosterone nor progesterone

379 mediated the change in oxidative condition after an acute increase in conspecific aggressive

380 behaviors. On the one hand, control and STI birds had similar testosterone concentrations;

381 thus, indicating that the difference in oxidative state observed between experimental groups is

382 not mediated by this steroid hormone. In line with our results, the relationship between

383 aggressive phenotypes and oxidative status was also independent of plasma testosterone

384 concentrations in both male and female wild-caught lizards (Isaksson et al., 2011). On the

385 other hand, we found that male, but not female, STI birds had higher progesterone

386 concentrations than controls; yet, our data suggests that aggressive behavior, oxidative status

387 and progesterone are not interrelated for this species: females suffered a much stronger STI-

388 induced decrease in OXY than males (see below), in neither sex there was a relationship

389 between aggressive behavior and progesterone concentrations, and we found no correlation

390 between OXY and progesterone in female and male STI horneros (Pearson's correlation;

$391 \mathrm{R}_{\text {female }}=-0.48, \mathrm{p}$-value $=0.19 ; \mathrm{R}_{\text {male }}=0.27$, $\mathrm{p}$-value $=0.29$ ).

392 The effect that acute conspecific aggressive behaviors had on OXY concentrations

393 was much stronger in females than in males (i.e., STI females had $40 \%$ lower OXY

394 concentrations than control females, whereas STI males had only 6\% lower OXY

395 concentrations than controls). This sex difference could be given by a differential re-

396 allocation of antioxidants to protect: gametes (Rojas Mora et al., 2017), the developing

397 embryo (see above; Shantz et al., 1999; Surai et al., 2001; Parolini et al. 2017), and/or those

398 tissues with the greatest oxygen consumption (e.g., muscles; Ji 2008). Sex differences could

399 also be explained by other physiological pathways besides an increase in activity levels (see

4004.3 for further discussion). The implications of these sex differences in non-enzymatic

401 antioxidant depletion will depend on the life-history stage that the individuals are in 
402 (Speakman et al., 2015). Repeated aggressive interactions experienced by females during egg

403 laying may come at a cost to the deposition of substances into the egg that could affect their

404 fitness. For example, an exercised group of female zebra finches (Taeniopygia guttata) that

405 had poorer oxidative status than non-exercised birds after flight training, decreased the

406 deposition of antioxidants (i.e., lutein) into the egg yolk (Skrip et al., 2016). However, birds

407 can also recover their circulating non-enzymatic antioxidant capacity by consuming

408 antioxidant-rich foods to avoid negative fitness consequences (e.g., Scrip \& McWilliams,

409 2016). Hence, to understand if aggression-induced perturbations in the oxidative status of an

410 individual have long-lasting functional or fitness consequences, future studies should ideally

411 measure the oxidative condition of an individual not only immediately after facing aggressive

412 interactions but also over repeated interactions, in relation to fitness traits and across different

413 life-history stages.

414 To the best of our knowledge this is the first study that demonstrates an effect of an 415 acute increase in aggressive behavior (i.e., 20 minutes) on the oxidative status in wild 416 animals. To date, only two other studies investigated the relationship between aggressive 417 behavior and oxidative status by looking at the correlation between the personality trait 418 "aggression" (i.e., over a long-term scale) and the oxidative status of a selected line of mice 419 (Costantini et al., 2008) and white skinks (Isaksson et al., 2011). Interestingly, these two 420 studies support our findings: they also found a relationship between aggression and OXY 421 concentrations, but not with ROMs as previously suggested. Altogether, this suggests that 422 aggression has both short- and long-term consequences on the oxidative status of an 423 individual, and that this occurs through a change in non-enzymatic antioxidants. On a broader 424 scale, these studies show that the relationship between aggression and oxidative status is 425 present across different vertebrate groups (i.e., reptiles, mammals and birds).

4.3. Relationships between acute conspecific aggressive behavior and the concentrations of oxidative markers, testosterone and progesterone

429 Whereas more aggressive females had lower levels of OXY than less aggressive females, no 430 such relationship was found in males. There was also no relationship between aggressive 431 behavior and testosterone or progesterone concentrations in either sex. Together with the 432 previous results, this indicates that mechanisms other than testosterone and progesterone 433 determine the oxidative condition of males and females after conspecific aggressive 434 interactions.

435 Engaging in aggressive interactions during the nest-building period could also have 436 led to an increase in another steroid hormone: glucocorticoids. An increase in glucocorticoid 437 concentrations can occur through an activation of the hypothalamic-pituitary-adrenal axis to 438 help an individual recover and survive an immediate challenge (Sapolsky et al., 2000). An 
439 increase in the concentration of this other steroid hormone after aggressive interactions has

440 been reported both in seasonally territorial (e.g., Charlier et al., 2009) and year-round

441 territorial vertebrates (Landys et al., 2010; Ros et al., 2014). Further, a direct link between

442 glucocorticoids and oxidative state has previously been reported (e.g., Haussmann \&

443 Marchetto 2010; Costantini et al., 2011; Haussmannn et al., 2012; Majer et al., 2019), and

444 females seem to be more susceptible to the effects of glucocorticoids on oxidative stress than

445 males (reviewed by Costantini et al., 2011, but see Ouyang et al., 2016). Hence, the

446 interaction between glucocorticoids and the redox system may provide an alternative

447 explanation of our results: aggression may lower OXY concentrations directly because of an

448 increased metabolism, or indirectly via an increase in glucocorticoid concentrations, or both.

\section{5. Conclusions}

450 Our study provides the first evidence that an acute increase in aggressive behavior perturbates 451 the oxidative state of female and male wild birds. Our study also suggests that the 452 mechanisms underlying these changes differ between sexes. Until now, the effect of 453 aggression on the oxidative state was investigated in the framework of personality traits 454 (Costantini et al. 2008; Isaksson et al. 2011). Altogether, the existing studies suggest that 455 aggressive behaviors performed over both short and long time scales have direct 456 consequences on the oxidative status of an individual. Remarkably, by generating a change in 457 non-enzymatic antioxidants and not in the concentration of pro-oxidants as frequently 458 suggested. Given that perturbations in the oxidative status can shape central life history traits 459 such as reproduction and longevity, the oxidative state of an individual might be a key 460 physiological mechanism underlying different life-history strategies in individuals that show 461 different levels of aggression.

\section{$462 \quad$ Ethical statement}

463 The experimental procedures were approved by the Ethics Committee of Animal 464 Experimentation (CEUA) of the Facultad de Ciencias, Universidad de la República, Uruguay 465 (University of the Republic of Uruguay). Protocol number 186, file 2400-11000090-16.

\section{Competing interests}

467 We declare no competing interests. 


\section{Funding}

469 We acknowledge funding and support from the International Max Planck Research School

470 (IMPRS) for Organismal Biology, and by Idea Wild that provided field equipment.

\section{Acknowledgements}

472 We thank Enzo Cavalli and Ernesto Guedes for their assistance in the field, and Monika

473 Trappschuh for conducting the hormone analysis. We also thank the members from the

474 Ethology Lab at the Universidad de la República in Montevideo and especially to Bettina

475 Tassino for their support. Also, we thank all the "INIA Las Brujas" staff for providing us with

476 accommodation and equipment during fieldwork. Furthermore, we are thankful to Juan Carlos

477 Reboreda and the Ecology and Behaviour lab (LEyCA) from the University of Buenos Aires

478 for the logistic support. We also thank Michaela Hau and Manfred Gahr for their valuable

479 support. We thank Laurie O'Neil and Shane McPherson for helping us with the English proof

480 of the manuscript. We are grateful to Stefania Casagrande, Michaela Hau, Wolfgang

481 Goymann and Scott McWilliams for valuable discussions about the study and constructive

482 criticism on previous versions of the manuscript. Lastly, we would like to thank the two

483 anonymous reviewers for constructive comments that helped improving this article. 


\section{References}

485

486 Adreani, N.M., Goymann, W. \& Mentesana, L. (2018) Not one hormone or another:

487 Aggression differentially affects progesterone and testosterone in a South American ovenbird.

488 Hormones and Behavior, 105, 104-109.

489

490 Alonso-Alvarez, C., Bertrand, S., Faivre, B., Chastel, O., \& Sorci, G. (2007). Testosterone

491 and oxidative stress: the oxidation handicap hypothesis. Proceedings of the Royal Society of

London B: Biological Sciences, 274(1611).

493

494

Apfelbeck, B., \& Goymann, W. (2011). Ignoring the challenge? Male black redstarts

495 (Phoenicurus ochruros) do not increase testosterone levels during territorial conflicts but they

496 do so in response to gonadotropin-releasing hormone. Proceedings. Biological Sciences,

497 278(1722), 3233-3242.

498

Araya-Ajoy, Y.G. \& Dingemanse, N.J. (2013) Characterizing behavioural "characters": an

500 evolutionary framework. Proceedings of the Royal Society of London B: Biological Sciences, 281, 20132645-20132645.

502

503 Beckman, K. B., \& Ames, B. N. (1998). The Free Radical Theory of Aging Matures.

504 Physiological Reviews, 78(2).

505

506 Bottje, W. (2015). Mitochondrial Physiology. Sturkie's Avian Physiology, 39-51.

507

508 Buchanan, K. L., Evans, M. R., Goldsmith, A. R., Bryant, D. M. \& Rowe, L. V. (2001).

509 Testosterone influences basal metabolic rate in male house sparrows: a new cost of 510 dominance signalling? Proceedings of the Royal Society of London Series B: Biological 511 Sciences 268, 1337-1344.

512

513 Casagrande S., Dijkstra C., Tagliavini J., Goerlich V. C.\& Groothuis T. G. G. G. (2011). 514 Differential effects of testosterone, dihydrotestosterone and estradiol on carotenoid deposition 515 in an avian sexually selected signal. Journal of Comparative Physiology. 197, 1-13.

517 Casagrande, S. \& Hau, M. (2018). Enzymatic antioxidants but not baseline glucocorticoids 518 mediate the reproduction-survival trade-off in a wild bird. Proceedings of the Royal Society 519 of London B: Biological Sciences, 285: 20182141. 
521 Chainy, G. B. N., Samantaray, S., \& Samanta, L. (2009). Testosterone-induced changes in

522 testicular antioxidant system. Andrologia, 29, 343-9.

523

524 Charlier, T. D., Underhill, C., Hammond, G. L., \& Soma, K. K. (2009). Effects of aggressive

525 encounters on plasma corticosteroid-binding globulin and its ligands in white-crowned

526 sparrows. Hormones and Behavior, 56, 339-47.

527

528

529

530 Cooper-Mullin, C., \& McWilliams, S. R. (2016). The role of the antioxidant system during

531 intense endurance exercise: lessons from migrating birds. The Journal of Experimental

532 Biology, 219(23), 3684-3695.

533

534 Costantini, D. (2008). Oxidative stress in ecology and evolution: lessons from avian studies.

535 Ecology Letters, 11(11), 1238-1251.

536

537 Costantini, D. (2019). Understanding diversity in oxidative status and oxidative stress: the

538 opportunities and challenges ahead. Journal of Experimental Biology, 222(13), jeb194688.

539

540 Costantini, D., Carere, C., Caramaschi, D., \& Koolhaas, J. M. (2008). Aggressive and non541 aggressive personalities differ in oxidative status in selected lines of mice (Mus musculus).

542 Biology Letters, 4(1).

543

544 Costantini, D., Casagrande, S., De Filippis, S., Brambilla, G., Fanfani, A., Tagliavini, J., \&

545 Dell'Omo, G. (2006). Correlates of oxidative stress in wild kestrel nestlings (Falco 546 tinnunculus). Journal of Comparative Physiology B, 176(4), 329-337.

547

548 Costantini, D., Marasco, V., \& Møller, A. P. (2011). A meta-analysis of glucocorticoids as 549 modulators of oxidative stress in vertebrates. Journal of Comparative Physiology B, 181(4), $550 \quad 447-456$.

551

552 Costantini, D., Monaghan, P., \& Metcalfe, N. B. (2011). Biochemical integration of blood 553 redox state in captive zebra finches (Taeniopygia guttata). The Journal of Experimental 554 Biology, 214(7), 1148-1152.

555 
556 Demas, G. E., Cooper, M. A., Albers, H.E., \& Soma, K.K. (2007). Novel mechanisms

557 underlying neuroendocrine regulation of aggression: a synthesis of rodent, avian, and primate

558 studies.

559

560 Diniz, P., Ribeiro, P. H. L., Rech, G. S., \& Macedo, R. H. (2016). Monochromatism, cryptic

561 sexual dimorphism and lack of assortative mating in the Rufous Hornero, Furnarius rufus

562 albogularis. Emu - Austral Ornithology, 116(3), 294-300.

563

564 Diniz, P., da Silva Júnior, E. F., Webster, M. S., \& Macedo, R. H. (2018). Duetting behavior

565 in a Neotropical ovenbird: sexual and seasonal variation and adaptive signaling

566 functions. Journal of Avian Biology.

567

568 Duckworth, R. A. (2006). Aggressive behaviour affects selection on morphology by 569 influencing settlement patterns in a passerine bird. Proceedings of the Royal Society of 570 London B: Biological Sciences, 273(1595).

571

572 Finkel, T., \& Holbrook, N. J. (2000). Oxidants, oxidative stress and the biology of ageing.

573 Nature, 408(6809), 239-247.

574

575 Fraga, R. M. (1980). The breeding of Rufous Horneros (Furnarius rufus). The Condor, 82(1), $57658-68$.

577

578 Freeman, B. G., Montgomery, G. A., \& Schluter, D. (2017). Evolution and plasticity:

579 Divergence of song discrimination is faster in birds with innate song than in song learners in

580 Neotropical passerine birds. Evolution, 71(9), 2230-2242.

581

582 Gavrilova-Jordan, L. \& Price, T. (2007). Actions of Steroids in Mitochondria. Seminars in 583 Reproductive Medicine, 25(3), 154-164.

584

585 Gelman, A., \& Hill, J. (2007). Data analysis using regression and multilevel/hierarchical 586 models.

588 Gill, S. A., Alfson, E. D., \& Hau, M. (2007). Context matters: female aggression and 589 testosterone in a year-round territorial neotropical songbird (Thryothorus leucotis).

590 Proceedings of the Royal Society B: Biological Sciences 274(1622): 2187-2194. 
592 Goymann, W., Landys, M. M., \& Wingfield, J. C. (2007). Distinguishing seasonal androgen

593 responses from male-male androgen responsiveness-revisiting the Challenge Hypothesis.

594 Hormones and Behavior, 51(4), 463-476.

595

596 Goymann, W., Wittenzellner, A., Schwabl, I., \& Makomba, M. (2008). Progesterone

597 modulates aggression in sex-role reversed female African black coucals. Proceedings of the

598 Royal Society of London B: Biological Sciences, 275(1638).

599

600 Halifeoglu, I., Karatas, F., Canatan, H., Colak, R., \& Karadas, E. (2003). Investigation of

601 antioxidant vitamins (A, E and C) and selenium levels in chickens receiving estrogen or

602 testosterone. Cell Biochemistry and Function, 21(2), 133-136.

603

604 Hau, M. (2007). Regulation of male traits by testosterone: implications for the evolution of 605 vertebrate life histories. Bioessays, 29, 133-144.

606

607 Haussmann, M. F., \& Marchetto, N. M. (2010). Telomeres: Linking stress and survival, 608 ecology and evolution. Current Zoology, 56(6), 714-727.

609

610 Haussmann, M. F., Longenecker, A. S., Marchetto, N. M., Juliano, S. A., \& Bowden, R. M. 611 (2012). Embryonic exposure to corticosterone modifies the juvenile stress response, oxidative 612 stress and telomere length. Proceedings of the Royal Society of London B: Biological 613 Sciences, 279(1732).

614

615 Hernandez, J. M., Fedele, M. J., \& Farrell, P. A. (2000). Time course evaluation of protein 616 synthesis and glucose uptake after acute resistance exercise in rats. Journal of Applied 617 Physiology, 88(3), 1142-1149.

618

619 Hirschenhauser, K., \& Oliveira, R. F. (2006). Social modulation of androgens in male 620 vertebrates: meta-analyses of the challenge hypothesis. Animal Behaviour, 71(2), 265-277. evolutionary perspective. Australian Journal of Zoology, 59(6), 369.

624 
629

630

631

632

633

634

635

636

637

638

639

640

641

642

643

644

645

646

647

648

649

650

651

652

653

654

655

656

657

658

659

660

661

662

663

664

665

Itagaki, T., Shimizu, I., Cheng, X., Yuan, Y., Oshio, A., Tamaki, K., Fukuno, H., Honda, H., Okamura, Y., \& Ito, S. (2005). Opposing effects of oestradiol and progesterone on intracellular pathways and activation processes in the oxidative stress-induced activation of cultured rat hepatic stellate cells. Gut, 12:1782-1789.

Ji, L. L. (2008). Modulation of skeletal muscle antioxidant defense by exercise: Role of redox signaling. Free Radical Biology and Medicine, 44(2), 142-152.

Koch, R. E., Josefson, C. C., \& Hill, G. E. (2016). Mitochondrial function, ornamentation, and immunocompetence. Biological Reviews, 92(3), 1459-1474.

Korner-Nievergelt, F., Roth, T., von Felten, S., Guélat, J., Almasi, B., \& Korner-Nievergelt, P. (2015). Bayesian data analysis in ecology using linear models with R, BUGS, and Stan.

Laje, R., \& Mindlin, G. B. (2003). Highly structured duets in the song of the South American hornero. Physical review letters, 91(25), 258104.

Landys, M. M., Goymann, W., Schwabl, I., Trapschuh, M., \& Slagsvold, T. (2010). Impact of season and social challenge on testosterone and corticosterone levels in a year-round territorial bird. Hormones and Behavior, 58(2),317-325.

Leeuwenburgh, C., \& Heinecke, J. (2001). Oxidative Stress and Antioxidants in Exercise. Current Medicinal Chemistry, 8(7), 829-838.

Majer, A. D., Fasanello, V. J., Tindle, K., Frenz, B. J., Ziur, A. D., Fischer, C. P., Fletcher, K. L., Seecof, K. L., Gronsky, S., Vasallo, B. G., Reed, W. L., Paitz, R. T., Stier, A., \& Haussmann, M. F. (2019). Is there an oxidative cost of acute stress? Characterization, implication of glucocorticoids and modulation by prior stress experience. Proceedings of the Royal Society of London B: Biological Sciences, 286.

Marler C. A. \& Moore M (1989). Time and Energy Costs of Aggression in TestosteroneImplanted Free-Living Male Mountain Spiny Lizards (Sceloporus jarrovi). Physiological Zoology, 62(6), 1334-1350.

Massoni, V., Reboreda, J. C., López, G. C., \& Aldatz, M. F. (2012). High coordination and equitable parental effort in the Rufous Hornero. The Condor, 114(3), 564-570. 
666 McGlothlin, J. W., Jawor, J. M., Greives, T. J., Casto, J. M., Phillips, J. L., \& Ketterson, E. D.

667 (2008). Hormones and honest signals: males with larger ornaments elevate testosterone more

668 when challenged. Journal of Evolutionary Biology, 21(1), 39-48.

669

670

Mentesana, L., Isaksson, C., Goymann, W., Andersson, M. N., Trappschuh, M., and Hau, M.

671 2019. Female variation in allocation of steroid hormones, antioxidants and fatty acids: a multilevel analysis in a wild passerine bird. Journal of Avian Biology, 50(1).

673

675 (2000). Carotenoid-dependent signals: indicators of foraging efficiency, immunocompetence 676 or detoxification ability? Avian Poultry Biol. Rev., 11, 137-159.

677

678 Monaghan, P., Metcalfe, N. B., \& Torres, R. (2009). Oxidative stress as a mediator of life 679 history trade-offs: mechanisms, measurements and interpretation. Ecology Letters, 12(1), 75680 92.

681

Nilsson, J.-Å., \& Råberg, L. (2001). The resting metabolic cost of egg laying and nestling 683 feeding in great tits. Oecologia, 128(2), 187-192.

684

685

Ouyang, J. Q., Lendvai, Á. Z., Moore, I. T., Bonier, F. \& Haussmann, M. F. (2016). Do Hormones, Telomere Lengths, and Oxidative Stress form an Integrated Phenotype? A Case Study in Free-Living Tree Swallows. Integrative and Comparative Biology, 56, 138-145. oxidative damage in gull hatchlings. Royal Society Open Science, 4.

692

693 Powers, S. K., \& Jackson, M. J. (2008). Exercise-Induced Oxidative Stress: Cellular

694 Mechanisms and Impact on Muscle Force Production. Physiological Reviews, 88(4).

695

696 R Core Team 2013. R: A language and environment for statistical computing. R Foundation 697 for Statistical Computing, Vienna, Austria. URL http://www.R-project.org/. in press. 
703 Ros, A. F. H., Vullioud, P., Bruintjes, R., Vallat, A., Bshary, R. (2014). Intra- and

704 interspecific challenges modulate cortisol but not androgen levels in a year-round territorial

705 damselfish. Journal of Experimental Biology, 217,1768-74.

706

707

Rosvall, K. A. (2008). Sexual selection on aggressiveness in females: evidence from an

708 experimental test with tree swallows. Animal Behaviour, 75(5), 1603-1610.

709

710

Royle, N. J., Surai, P. F., Mccartney, R. J., \& Speake, B. K. (1999). Parental investment and egg yolk lipid composition in gulls. Functional Ecology, 13(3), 298-306.

712

713

Salin, K., Auer, S. K., Rudolf, A. M., Anderson, G. J., Cairns, A. G., Mullen, W., Hartley, R.

714 C., Selman, C., \& Metcalfe, N. B. (2015). Individuals with higher metabolic rates have lower levels of reactive oxygen species in vivo. Biology Letters, 11:20150538. stress responses? Integrating permissive, suppressive, stimulatory, and preparative actions. Endocrine Reviews, 21(1), 55-89. oxidative stress and condition-dependent sexual signals. Proceedings of the Royal Society of London B: Biological Sciences, 266(1414). organisms-to-ecology primer for ornithologists. Journal of Field Ornithology, 87(1), 1-20. carry-over effects work. Journal of Experimental Biology, 219(17). analysis. Behavioral Ecology, 19(2), 448-455.

735 Speakman, J. R. (2003). Oxidative phosphorylation, mitochondrial proton cycling, free736 radical production and aging. 14, 35-68. 
738 Speakman, J. R., Blount, J. D., Bronikowski, A. M., Buffenstein, R., Isaksson, C., Kirkwood,

739 T. B. L., et al. (2015). Oxidative stress and life histories: unresolved issues and current

740 needs. Ecology and Evolution. 5, 5745-5757.

741

742 Surai, P. F. (2002). Natural antioxidants in avian nutrition and reproduction. British Library

743 Cataloguing in Publication Data Poultry Environment Problems: A Guide to Solutions.

744

745 Surai, P. F., Speake, B. K., \& Sparks, N. H. C. (2001). Carotenoids in avian nutrition and 746 embryonic development. 1. Absorption, availability and levels in plasma and egg yolk. The

747 Journal of Poultry Science, 38(1), 1-27.

748

749 Villavicencio, C. P., Blas, J., \& Goymann, W. (2014). The number of life-history stages does 750 not influence the androgen responsiveness to male-male interactions: Sedentary and 751 migratory black redstarts (Phoenicurus ochruros) do not elevate testosterone in response to 752 simulated territorial intrusions. General and Comparative Endocrinology, 205, 159-165.

753

754 Welle S., Jozefowicz R., Forbes G, Griggs R. C. (1992). Effect of testosterone on metabolic 755 rate and body composition in normal men and men with muscular dystrophy. The Journal of 756 Clinical Endocrinology \& Metabolism, 74(2), 332-335,

757

758 Wikelski, M., Lynn, S., Breuner, J. C., Wingfield, J. C., \& Kenagy, G. J. (1999). Energy 759 metabolism, testosterone and corticosterone in white-crowned sparrows. Journal of 760 Comparative Physiology A: Sensory, Neural, and Behavioral Physiology, 185(5), 463-470.

762 Wingfield, J. C., \& Wada, M. (1989). Changes in plasma levels of testosterone during male763 male interactions in the song sparrow, Melospiza melodia: time course and specificity of 764 response. Journal of Comparative Physiology A, 166(2), 189-194.

766 Wingfield, J. C., Hegner, R. E., Dufty, A. M., \& Ball, G. F. (1990). The "Challenge 767 Hypothesis": Theoretical Implications for Patterns of Testosterone Secretion, Mating 768 Systems, and Breeding Strategies. The American Naturalist, 136(6), 829-846.

770 Wingfield, J. C. (1994). Regulation of Territorial Behavior in the Sedentary Song Sparrow, 771 Melospiza melodia morphna. Hormones and Behavior, 28(1), 1-15.

773 Wingfield, J. C., \& Hahn, T. P. (1994). Testosterone and territorial behaviour in sedentary 774 and migratory sparrows. Animal Behaviour, 47(1), 77-89. 
776 Woodley, S. K., \& Moore, M. C. (1999). Female territorial aggression and steroid hormones

777 in mountain spiny lizards. Animal Behaviour, 57(5), 1083-1089.

778

779 Zhu, X. D., Bonet, B., Knopp, R. H. (1997). 17ß-estradiol, progesterone, and testosterone

780 inversely modulate low- density lipoprotein oxidation and cytotoxicity in cultured placental

781 trophoblast and macrophages. American Journal of Obstetrics and Gynecology, 177, 196782209.

783 